\title{
Evaluation of a Commercial ELISA Kit (IDEXX) to Differentiate Al Virus-infected Poultry from Al- Vaccinated Poultry (DIVA)
}

http://dx.doi.org/10.1590/1516-635x160273-78

\section{-Author(s)}

Talazadeh $\mathrm{F}^{\mathrm{I}}$

Mayahi M"

Seifi M"II

Pourmehdi $\mathrm{M}^{\mathrm{N}}$

Assistant Professor, Department of Clinica Sciences, Faculty of Veterinary Medicine, Shahid Chamran University of Ahvaz, Iran

" Professor, Department of Clinical Sciences, Faculty of Veterinary Medicine, Shahid Chamran University of Ahvaz, Iran

III Professor, Department of Pathobiology, Faculty of Veterinary Medicine, Shahid Chamran University of Ahvaz, Iran

iv Assistant Professor, Department of Food Hygiene and Quality control, Faculty of Veterinary Medicine, Shahid Chamran University of Ahvaz, Iran

\section{Mail Adress}

Corresponding author e-mail address

F Talazadeh. Department of Clinical Sciences, Faculty of Veterinary Medicine, Shahid Chamran University of Ahvaz, Iran.

E-mail: ftalazade@gmail.com

\section{nKeywords}

Avian influenza virus (AIV), DIVA, commercial ELISA.

\begin{abstract}
Vaccination programs for the control of avian influenza (Al) in poultry have limitations due to the problem of differentiating between vaccinated and virus-infected birds. The use of Al vaccination in poultry would have greater worldwide acceptance if reliable tests that clearly discriminated between naturally infected and vaccinated-only animals (DIVA) were available. In this study, to differentiate avian influenza virus (AIV)-infected chickens vs. chickens immunized with inactivated avian influenza virus, a commercial ELISA Kit (IDEXX) was used. Using HI as the reference method, the sensitivity of the commercial ELISA kit was 100\% and its specificity was $100 \%$. In the present study, we demonstrated that, 3 weeks after infection or vaccination, sera from both infected and vaccinated groups were tested positive and the difference in mean optical density between vaccinated and challenged birds, as detected by a commercial ELISA kit (IDEXX), was very small. Therefore, this assay cannot distinguish infected from vaccinated poultry.
\end{abstract}

\section{INTRODUCTION}

Influenza viruses are segmented, negative-strand RNA viruses belonging to the family Orthomyxoviridae, and are divided in three types: A, B, and C - Wright et al. (2007). All three types of influenza virus are found in humans, but only the type $A$ viruses are found in poultry and are referred to as avian influenza (Al) viruses.

Type A influenza viruses are divided into subtypes based on the antigenic properties of the major surface glycoproteins: hemagglutin in (HA) and neuraminidase (NA) proteins. In total, 16 HA subtypes $(\mathrm{H} 1-\mathrm{H} 16)$ and nine neuraminidase subtypes (N1-N9) are currently described - Wright et al. (2007). H9N2 virus shares similar receptor binding epitopes with human influenza viruses, has a broader host range, and has been demonstrated to infect humans. In addition, H9N2 AIV infection in chickens is latent and easily overlooked, increasing its chance of infecting humans.

Although Al vaccines have been successful at providing protection against clinical signs and death among poultry - Garcia et al. (1998), Halvorson (2002) and Swayne (2001), they are not commonly used as part of programs for the control and eradication of LPAI or HPAI virus infections - Halvorson (2002). For a number of reasons, including the concerns with trade embargoes on poultry and poultry products, quarantine and elimination of infected flocks (i.e., stamping out or controlled marketing) are often preferred measures for controlling Al. An additional limitation is that traditional Al virus vaccines can interfere with serologic surveillance; hence, these vaccines induce antibodies that are indistinguishable from live virus infection, as determined by commercial enzyme-linked immunosorbent assays (ELISAs), and hemagglutination 
inhibition (HI) tests - Swayne (2003). Therefore, efforts have been focused on developing new serologic tools to allow differentiation of infected from vaccinated animals, commonly known as the DIVA strategy.

For international commerce of vaccinated birds, the DIVA strategy needs to not only discriminate between infected and vaccinated birds, but also to identify vaccinated birds that become infected with AIV. Several different DIVA strategies have been proposed - Suarez (2005), some of which require the use of appropriate vaccines and specific companion serologic discriminatory tests - Capua et al. ( 2004), Capua et al. (2003), Cattoli et al. ( 2006), Lee et al. ( 2004) and Tumpey et al. (2005). Such a strategy is important to provide assurance to trading partners that the poultry product is free from influenza virus contamination and can be safely traded. The DIVA strategy has been accepted by some countries in the European Union to monitor and provide assurance on the infection-free status of vaccinated poultry during an LPAI outbreak Capua \& Marangon (2003) and Suarez (2005).

One potential target for the DIVA strategy for avian influenza is the nonstructural (NS1) protein. The NS1 protein is encoded by the smallest gene segment of the influenza virus, segment 8 , and it has a critical role in preventing the induction of interferon in the host to allow efficient virus replication - Garcia-Sastre et al. (1998). The NS1 protein is a conserved protein amongst type $A$ influenza viruses, but it exists as two major subtypes, $A$ and $B$, that differ by about 35\% in nucleotide sequence - Suarez \& Perdue (1999). The NS1 protein is produced during influenza virus replication in infected cells - Birch-Machin et al. (1997) and Tumpey et al. (2005), but it is not packaged into the infectious viral particle, being a true nonstructural protein. Because most AIV vaccines are primarily chemically inactivated whole virus, vaccinated animals theoretically should not have an antibody response to the NS1 protein, but infected poultry should have an NS1 antibody response because it is produced at high levels in infected cells.

This NS1 DIVA approach was first proposed for equine influenza viruses, and purified NS1 protein has been used to detect antibodies against NS1 in serum samples from ponies and horses experimentally infected with influenza virus - Birch-Machin et al. (1997) and Ozaki et al. (2001). Serologic studies on chickens and turkeys using an ELISA with AIV NS1 protein produced in Escherichia coli as antigen indicated that infected birds showed higher levels of NS1 antibodies as compared to vaccinated birds - Tumpey et al. (2005). And Zhao et al. ( 2005).

\section{MATERIALS AND METHODS}

\section{Chickens}

A total of 300 day-old broiler chicks (Ross 308) were purchased. Birds were housed in three different rooms and raised under high health conditions.

\section{Virus}

The Al viruses used in this study derived from a virus isolate (A/Chicken/Iran/AH-1/06), subtype H9N2, from a broiler chicken flock in Ahvaz, with respiratory signs and mortality. These viruses were initially isolated in 10-day-old embryonated chicken eggs (ECE). They were subtyped by standard hemagglutination-inhibition and neuraminidase-inhibition tests with polyclonal chicken antisera. The viruses were passaged in ECE and the all antoic fluids were harvested and used as stock viruses for further analysis.

\section{Vaccines}

AIV vaccine consisting of inactivated commercial Al vaccines, subtypes H9N2.

\section{Experimental design}

Birds were divided into three equal groups (1 to 3). Chicks in groups 1 were subcutaneously vaccinated (day 21) in the back of the neck with an AIV H9N2 killed vaccine. Chicks in group 2 were intranasally infected (day 21) with $0.2 \mathrm{~mL}$ infectious all antoic fluid. Chicks in group 3 were kept as control group and did not receive vaccine or live virus. Serum was collected on day 42 and used in ELISA test design.

\section{Commercial ELISA}

A commercial ELISA assay was performed in U-bottomed 96-well microtiter plates with commercial ELISA kit) IDEXXCo.).

\section{Hemagglutination inhibition (HI) assay}

Beta procedure of hemagglutination-inhibition $(\mathrm{HI})$ assays was performed in U-bottomed 96-well microtiter plates with 5\% chicken erythrocytes - Spackman \& Pedersen (2008).

\section{RESULTS}

\section{Sensitivity and specificity of commercial ELISA kit (IDEXX)}

In order to evaluate the specificity and sensitivity of the commercial ELISA kit (IDEXX), 268 serum samples were collected from the experimental birds, including 90 samples from birds vaccinated with the inactivated 
vaccine, 90 samples from those experimentally infected with live H9N2 AIV by intranasal inoculation, and 88 samples from control birds.All180 serum samples of vaccinated chickens and infected chickens were positive by $\mathrm{HI}$. Using $\mathrm{HI}$ as the reference method, the sensitivity of the commercial ELISA kit was $100 \%$ and its specificity was $100 \%$.

\section{Results of experimental bird serum detection using a commercial ELISA kit (IDEXX)}

The collected sera were assayed using a commercial ELISA kit (IDEXX).Three weeks after infection or vaccination, sera of the infected and vaccinated groups tested positive by the commercial ELISA kit (IDEXX). Sera of all control chickens were negative in this assay (Table 1-1,1-2).

Table 1-1 : Results of the serum of control and challenge group in IDEXX.

\begin{tabular}{|c|c|c|c|}
\hline \multirow[t]{2}{*}{ IDEXX } & \multicolumn{2}{|c|}{ Status } & \multirow[t]{2}{*}{ Tota } \\
\hline & Challenge & Control & \\
\hline Positive & 90 & 0 & 90 \\
\hline Negative & 0 & 88 & 88 \\
\hline Total & 90 & 88 & 178 \\
\hline
\end{tabular}

Relative sensitivity $=(90 / 90) \times 100=100 \%$, Relative specificity $=(88 / 88) \times 100=100 \%$, Accuracy $=(90+88) / 178 \times 100=100 \%$, Kappa statistic $=1$,

Positive predictive value $=(90 / 90) \times 100=100 \%$,

Negative predictive value $=(88 / 88) \times 100=100 \%$, McNemar Test $(p>0.05)$

Table 1-2: Results of the serum of control and vaccinated group in IDEXX.

\begin{tabular}{lccc}
\hline \multirow{2}{*}{ IDEXX } & \multicolumn{2}{c}{ Status } & Total \\
\cline { 2 - 3 } & Vaccinated & Control & \\
\hline Positive & 90 & 0 & 90 \\
\hline Negative & 0 & 88 & 88 \\
\hline Total & 90 & 88 & 178 \\
\hline
\end{tabular}

Relative sensitivity $=(90 / 90) \times 100=100 \%$, Relative specificity $=(88 / 88) \times 100=100 \%$ ， Accuracy $=(90+88) / 178 \times 100=100 \%$, Kappa statistic $=1$ ，

Positive predictive value $=(90 / 90) \times 100=100 \%$,

Negative predictive value $=(88 / 88) \times 100=100 \%, \operatorname{McNemar}$ Test $(p>0.05)$

\section{DISCUSSION}

Because low pathogenic avian influenza (LPAI) virus infections among chickens and turkeys are often asymptomatic, the diagnosis requires serologic monitoring. Most poultry diagnostic laboratories prefer the serologic AGP test because of its simplicity and broad specificity for the detection of type A influenza virus infections among poultry. AGP antibodies recognize conserved nucleoprotein (NP) and matrix (M1) influenza virus proteins present in the sera of poultry exposed to Al virus and therefore can detect multiple subtypes of influenza A virus - Beard (1989). Other serologic tests for Al include ELISA, which also measures antibody responses to conserved internal proteins, and the subtype-specific HI assay - Office International des Epizooties (1996). However, these standard serologic tests for Al virus exposure do not differentiate between vaccinated and infected poultry when traditional vaccines are used. An improved serologic assay for Al virus infection could allow the identification of poultry that have been infected with Al virus, while it would correctly exclude animals that have been vaccinated with any subtype.

The diagnostic test described in this study used a conserved nonstructural protein that is not associated with virions, but that is expressed in large amounts in influenza virus-infected cells - Krug \& Etkind (1973), the NS1 protein. Following dilution of immune sera, the NS1 ELISA was an effective tool for the screening of poultry sera to detect Al virus infection. The NS1 protein, although a weak antigen in comparison with other influenza virus proteins, is remarkably conserved among type A influenza viruses - Suarez \& Perdue (1999), Buonagurio et al. (1986), Ludwig et al. (1991) and Treanor et al. (1989). Previous studies demonstrated that the NS1 protein can be detected in the sera of horses experimentally infected with the $\mathrm{H} 3$ subtype of influenza virus, but not in the sera of animals immunized with inactivated viruses - Birch-Machin et al. (1997) and Ozaki et al. (2001). The inactivated whole-virus equine vaccine used in the aforementioned studies was partially purified, thus removing residual NS1 protein that could be present in the infectious medium. This allowed the clear distinction between $\mathrm{H} 3$-vaccinated and $\mathrm{H} 3$-infected horses by using the NS1 protein as a differential marker - Birch-Machin et al. (1997) and Ozaki et al. (2001).

Birch-Machin et al. (1997) and Ozaki et al. (2001) described application an ELISA capable of differentiating AIV antibodies induced by vaccination from those induced by infection. Antibodies against the NS1 protein of AIV were considered the most useful marker of virus replication. Most sera of the infected chickens were positive, while all the sera of the vaccinated chickens tested negative in the NS1-ELISA as well as sera of the control chickens without previous exposure to AIV antigens either by vaccination or infection. 
The $\mathrm{HI}$ test has been used as a conventional method of diagnosis to detect antibodies against influenza viruses. However, the $\mathrm{HI}$ test does not differentiate antibodies induced by infection from those induced by vaccination. In theory, chickens immunized with an inactivated virus vaccine possess antibodies against primarily structural proteins of the virus, while chickens infected with LPAI virus produce antibodies against nonstructural AIV proteins as well. Thus, methods detecting antibodies against nonstructural AIV proteins could be used as a means of differentiation.

In the present study, we demonstrated that, by ELISA using $E$. coli expressed recombinant NS1 as antigen, anti-NS1 antibodies were detected only in the serum samples of experimentally infected chickens and not in the vaccinated chickens. This observation was also made by Tumpey et al. (2005). in a recent study - Tumpey et al. (2005). The results of the study of Tumpey et al. (2005) indicated that the use of a simple and specific ELISA for the sero diagnosis of infection with multiple LPAI virus subtypes can distinguish infected from vaccinated poultry. In that study, animals inoculated with inactivated gradient-purified vaccines had no sero conversion to positivity for antibodies against the NS1 protein, and animals vaccinated with commercial vaccines had low, but detectable, levels of NS1 antibodies - Tumpey et al. (2005). The use of a second ELISA with diluted sera identified a diagnostic test that results in seropositive for antibodies against the NS1 protein only in infected birds - Tumpey et al. (2005). For the field application phase of this study, serum samples were collected from vaccinated and infected poultry, diluted, and screened for anti-NS1 antibodies-Tumpey etal. (2005). Field sera from poultry that received commercial Al killed vaccines were found to possess antibodies against the Al virus, as measured by the standard agar gel precipitin (AGP) test, but they were negative by the NS1 ELISA - Tumpey et al. (2005). Conversely, diluted field sera from Al-infected poultry were positive for both AGP and NS1 antibodies. These results demonstrate the potential benefit of a simple, specific ELISA for anti-NS1 antibodies that may have diagnostic value for the poultry industries - Tumpey et al. (2005). This observation was also made by Zhao et al. in a recent study - Zhao et al. ( 2005). In study of Zhao et al. NS1-ELISA test was used in comparison with the $\mathrm{HI}$ test to detect antibodies againstNS1 in serum samples. In order to evaluate the specificity and sensitivity of the NS1-ELISA, 99 serum samples were collected from experimental chickens, including 72 samples from chickens vaccinated with an inactivated vaccine and 27samples from chickens experimentally infected with live H9N2 Al V via subcutaneous or intramuscular injection - Zhao et al. ( 2005). All99 serum samples were positive by HI. Using theNS1-ELISA, all 27 samples from infected chickens were tested positive, and out of the 72 vaccinated samples, 68 were tested negative and there maining four samples were weakly positive. Using $\mathrm{HI}$ as the reference method, the sensitivity of the NS1-ELISA was 100\% (27/27) and its specificity was $94.4 \%$ (68/72) - Zhao et al. ( 2005). In present study, sera of chicks experimentally infected with AIV and chicks vaccinated with a killed influenza vaccine were tested positive by the commercial ELISA kit (IDEXX). Sera of all control chickens were negative. In order to evaluate the specificity and sensitivity of the commercial ELISA kit (IDEXX), 268 serum samples were collected from the experimental chickens, including 90 samples from chickens vaccinated with an inactivated vaccine, 90 samples from chickens experimentally infected with live H9N2 AIV via intranasal inoculation, and 88 samples from chickens of the control group. The 180 serum samples of vaccinated chickens and infected chickens are all positive by $\mathrm{HI}$. Using $\mathrm{HI}$ as the reference method, the sensitivity of the commercial ELISA kit was $100 \%$ and its specificity was $100 \%$. In the present study, we demonstrated that, 3 weeks after infection or vaccination, sera of both infected and vaccinated groups were tested positive and the difference in mean optical density between vaccinated and challenged birds, as detected by a commercial ELISA kit (IDEXX), was very small. Therefore, this assay cannot distinguish infected from vaccinated poultry.

Abbreviations: $\mathrm{Al}=$ avian influenza; $\mathrm{AIV}=$ avian influenza virus; $b p=$ base pairs; $B S A=$ bovine serum albumin; ECE = embryonated chicken eggs; ELISA = enzyme-linked immunosorbent assay; HEPA = highefficiency particulate air; $\mathrm{HI}=$ hemagglutinin-inhibition; $\mathrm{HPAI}=$ high pathogenicity Al; IPTG = isopropyl-b-Dgalactopyranoside; LPAI = low pathogenicity Al; NS1 = nonstructural protein; $\mathrm{OD}=$ optical density; $R T-P C R=$ reverse transcription-polymerase chain reaction; $\mathrm{SPF}=$ specific-pathogen-free; TBS = Tris-buffered saline; TMB $=3,3,5,5$-tetramethyl benzidine.

\section{REFERENCES}

Beard CW.Serological procedures, In H. Friedman (ed.) A laboratory manual for the isolation and identification of avian pathotypes. Kendall-Hunt Publishing, Dubuque, lowa.1989; 192-200.

Birch-Machin I, Rowan A, Pick J, Mumford J and BinnsM. Expression of the nonstructural protein NS1 of equine influenza A virus: detection of anti-NS1 antibody in post infection equine sera. J. Virol. Methods1997;65:255-263. 
Buonagurio DA, Nakada S, Parvin J D, Krystal M, Palese P and Fitch W M. Evolution of human influenza A viruses over 50 years: rapid, uniform rate of change in NS gene. Science1986; 232:980-982.

Capua I, Cattoli G and Marangon S.DIVA-a vaccination strategy enabling the detection of field exposure to avian influenza. Dev. Biol. (Basel) 2004; 119:229-233

Capua I, and Marangon S. Vaccination policy applied for the control of avian influenza in Italy. Dev. Biol. (Basel) 2003;114:213-219.

Capua I, Terregino C , Cattoli G, Mutinelli F. and Rodriguez J F. Development of a DIVA (Differentiating Infected from Vaccinated Animals) strategy using a vaccine containing a heterologous neuraminidase for the control of avian influenza.AvianPathol.2003; 32:47-55.

Cattoli G, Milani A, Bettini F, Serena Beato M, MancinM, Terregino $\mathrm{C}$ and Capua I. Development and validation of an anti-N3 indirect immunofluorescent antibody test to be used as a companion diagnostic test in the framework of a "DIVA" vaccination strategy for avian influenza infections in poultry.AvianPathol. 2006;35:154-159.

Wright PE, Neumann $G$ and Kawaoka Y. Orthomyxoviruses In: Fields Virology, fifth ed. Lippincott, Williams and Wilkins, Philadelphia, PA. 2007;1692-1731.

Garcia-Sastre A, Egorov A, Matassov D,Brandt S, Levy D E, Durbin J E, Palese $P$ and Muster T. Influenza A virus lacking the NS1 gene replicates in interferon-deficient systems. Virology. 1998;252:324-330.

Garcia A,Hector J , Srivastava D K , Jayawardene D A, Wehr D R and WebsterR G.Efficacy of inactivated H5N2 influenza vaccines against lethal A/Chicken/Queretaro/19/95 infection. Avian Dis. 1998; 42:248256.

Halvorson DA. The control of H5 or H7 mildly pathogenic avian influenza a role for inactivated vaccine.AvianPathol.2002;31:5-12.

Krug R M, and Etkind P R. Cytoplasmic and nuclear virus-specific proteins in influenza virus-infected MDCK cells. Virology 1973; 56:334-348.

Lee C W, Senne D A and Suarez D L.Generation of reassortant influenza vaccines by reverse genetics that allows utilization of a DIVA (Differentiating Infected from Vaccinated Animals) strategy for the control of avian influenza. Vaccine 2004;22:3175-3181.

Ludwig S, Schultz U, Mandler J, Fitch WM and Scholtissek C. Phylogenic relationship of the nonstructural (NS) genes of influenza A viruses. Virology1991;183:566-577.

Office International des Epizooties. Highly pathogenic avian influenza (fowl plague), In Manual of standards for diagnostic tests and vaccines, vol. 3. Office International des Epizooties, Paris, France. 1996;155-160.

Ozaki H, Sugiura T, Sugita S, ImagawaHand Kida H. Detection of antibodies to the nonstructural protein (NS1) of influenza A virus allows distinction between vaccinated and infected horses. Vet. Microbiol. 2001;82:111119.
Spackman E and Pedersen J C. Avian Influenza Virus. 2008:8 :53-65.

Suarez DL. Overview of avian influenza DIVA test strategies. Biologicals 2005:33:221-226.

Suarez D L, and Perdue ML. Multiple alignment comparison of the nonstructural genes of influenza A viruses. Virus Res. 1999;54:59-69

Suarez DL, Perdue ML. Multiple alignment comparison of the non-structural genes of influenza A viruses. Virus Research 1999;54:59-69.

Suarez DL. Overview of avian influenza DIVA test strategies. Biologicals 2005;33:221-226.

Swayne D E, Beck J R, Perdue M L and Beard C W. Efficacy of vaccines in chickens against highly pathogenic Hong Kong H5N1 avian influenza. Avian Dis. 2001;45:355-365

Swayne D E. Vaccines for list A poultry diseases: emphasis on avian influenza. Dev. Biologics (Basel) 2003;114:201-212.

Swayne DE, Beck JR, Perdue ML, Beard CW. Efficacy of vaccines in chickens against highly pathogenic Hong Kong H5N1 avian influenza. Avian Diseases 2001;45:355-365

Swayne DE. Vaccines for list A poultry diseases: emphasis on avian influenza. Development Biology 2003;114:201-212.

Treanor JJ, Synder M H ,London WT, Murphy BR. The B allele of the NS gene of avian influenza viruses, but not the A allele, attenuate a human influenza A virus for squirrel monkeys. Virology 1989;171:1-9.

Treanor JJ, Synder M H, LondonWT and Murphy BR. The B allele of the NS gene of avian influenza viruses, but not the $A$ allele, attenuate a human influenza A virus for squirrel monkeys. Virology 1989;171:1-9.

Tumpey TM, Alvarez R, Swayne DE, Suarez DL.Diagnostic approach for differentiating infected from vaccinated poultry on the basis of antibodies to NS1, the nonstructural protein of influenza A virus. Journal Clinical Microbiology 2005;43:676-683.

Tumpey TM, Alvarez R, Swayne D E and Suarez D L.Diagnostic approach for differentiating infected from vaccinated poultry on the basis of antibodies to NS1, the nonstructural protein of influenza A virus. J. Clin. Microbiol.2005;43:676-683.

Wright PE, Neumann G, Kawaoka Y. Orthomyxoviruses In: Knipe DM Howley PM. Fields virology. $5^{\text {th }}$ ed. Lippincott: Williams and Wilkins, Philadelphia,2007. p.1692-1731.

Zhao S, Jin M, Li H, TanY, Wang G, Zhang R, Chen H. Detection of antibodies to the nonstructural protein (NS1) of avian influenza viruses allows distinction between vaccinated and infected chickens. Avian Diseaes 2005:49:488-493.

Zhao S, Jin M, Li H, TanY, Wang G, Zhang R, and ChenH. Detection of antibodies to the nonstructural protein (NS1) of avian influenza viruses allows distinction between vaccinated and infected chickens. Avian Dis. 2005;49:488-493. 
